\title{
Caracterización técnica y ambiental de la producción agropecuaria tradicional de Carhuaz, Áncash, Perú
}

\author{
Technical and environmental characterization of traditional farming of \\ Carhuaz, Ancash, Peru \\ Juan Barreto Rodríguez ${ }^{1}$, Manuel Canto Sáenz ${ }^{2}$, Alberto Julca Otiniano² y \\ FÉlix Camarena MaYta ${ }^{2}$
}

\section{RESUMEN}

La firma de los Tratados de Libre Comercio (TLC) entre el Perú y varios países del mundo, ha dinamizado la economía rural, afectando no solo a los medianos productores, sino también a los pequeños, provocando cambios en la estructura productiva y en el manejo de cultivos y crianzas. En este nuevo contexto se hace una descripción de las características técnicas y tecnológicas de los sistemas de Producción Agropecuaria Tradicional (PAT) de Carhuaz y de las condiciones topográficas y edáfico-climáticas del lugar. Para ello se aplicaron encuestas a una muestra representativa de 339 jefes de familia de los cinco distritos involucrados: Yúngar, Anta, Marcará, Carhuaz y Ataquero. Entre los aspectos resaltantes encontrados se tiene la predominancia del minifundio, la baja disponibilidad de agua de riego, el uso de bajos niveles tecnológicos, bajo rendimiento de los cultivos, la ganadería extensiva, etc. La información servirá de línea de base para futuros trabajos de investigación y para emprender las innovaciones en el manejo de los sistemas PAT, orientadas a mejorar rendimientos y consecuentemente los ingresos, la nutrición y el bienestar de la población.

Palabras clave: agricultura tradicional; sistemas de producción agropecuaria; minifundio.

\begin{abstract}
The signing of the Free Trade Agreement (FTA) between Peru and several countries around the world, has boosted rural economy, affecting not only medium-sized producers, but also to small, causing changes in culture cells and in the management of crops and breeding. In this context, is made a description of the technical and technological characteristics of traditional farming of Carhuaz; also the topographical, edafic and climatic conditions of this place. For this purpose we applied surveys to a representative sample of 339 heads of family, of five districts involved: Yungar, Anta, Marcara, Carhuaz, and Ataquero. Among the salient points found is the predominance of the smallholding, the low availability of irrigation water, the use of a low-technology, low yield of crops, cattle ranching, etc. These data will serve as a baseline basis for future works of research and to undertake innovations in management of traditional farming systems, aimed at improving returns and consequently income, nutrition, and well-being of the population.
\end{abstract}

\footnotetext{
1 Universidad Nacional «Santiago Antúnez de Mayolo». Huaraz, Perú.

2 Universidad Nacional Agraria La Molina. Lima, Perú.
} 
Keywords: traditional agriculture; agricultural production systems; smallholding.

\section{ICHIKLLACHAW}

Piruwan wakin waqta suyukunawan (TLC) hutishqa huwirmayashqan, tuna ikunumiyankunata kawararatsishqa, tsay pullan mikuykuna wachatsiqkunallatatsu, mana tsayqa ichikkunatawanmi, tsay istruktura pruduktiba nishqanta tikratsishqa, hina murukuywan ashma waatay apayninchaw pismi. Kay huklaaya kaq kuntixtuqa nin llapan tiknikakunawan tiknuluhiyakuna diskripsiyunta unay murukuykunapa wachayninta (PAT) Qarwaspa patsanchaw nishqantam, llapan tsaykunata imanawpis kanqantam. Tsaypaqmi tapukuykunanata rurakashqa, taytakunapa kastakunapaq, kayta 339 pushaq kastakunapaqmi rurakashqa, pitsqaq Qarwaspa distritunkunachaw: Yunkar, Anta, Markara, Qarwas, Ataqirupis. Kaychaw alli rikakuynin kashqa minihundiyukunachawmi, pishiq yaku parquyninchaw, ichik nibilkunapa tiknuluhiyankunachaw, murukuykunapa mana alli wachay yarquynin, ashmakunapa mana alli waataynin. Kay musyapakuyqa allim wakin pikunapis ashipakuq ruraqkunapaqmi, PATta alliyaatiyananpaq, tsaynawpa alliyaananpaq, mushuq yaykuykuna wiñananpaq, hina llapankunapa markankunachaw mikuyninkuna alliyaananpaq.

Pushaq shimikuna: ñawpa murukuykuna; chakrapawan ashma waataykunapawan wachayninkuna; minihundiyu.

\section{INTRODUCCIÓN}

La producción agropecuaria tradicional (agricultura familiar, con bajo nivel tecnológico) sigue siendo uno de los sectores más relevantes de la economía local y regional, ya que produce alimentos de primera necesidad para la población rural y para las poblaciones de las principales ciudades de la zona. Sin embargo, en el contexto actual, no hay información sobre los sistemas de producción agropecuaria tradicional (PAT). En tal virtud, en el marco de esa realidad, se ha materializado este trabajo con la finalidad de identificar algunos aspectos críticos sobre los cuales se pueda incidir para mejorar la productividad de los cultivos y crianzas y con ello la nutrición e ingresos de la población. Los campesinos producen en condiciones de clima y topografía difíciles, con muchos problemas y limitaciones, es decir, en laderas de diferente pendiente y, en muchos casos, con ocurrencia de heladas, granizadas, sequía, plagas y enfermedades que afectan la producción. Echenique (2006), en un estudio realizado en varios países de Latinoamérica, reporta que los predios de la agricultura familiar se ubican en los rangos de tamaños más pequeños de la tenencia agrícola y mayoritariamente en áreas con menor potencial de recursos naturales (suelo y agua). Así mismo menciona que, además de las restricciones en el acceso a la tierra, las explotaciones familiares disponen generalmente de poco capital de trabajo y de reducidas capacidades para acumular excedentes. Así mismo, están sometidas a restricciones de acceso al financiamiento de las instituciones formales.

$\mathrm{Al}$ respecto, Naredo (1996) menciona que tanto las plantaciones como las rotaciones de cultivos, se ajustan a las limitaciones edafo-climáticas de su territorio. En ese mismo sentido, Montalba (2004) reporta que los sistemas agrarios tradicionales, han debido adaptarse a los límites marcados por las características de suelo y clima de cada zona, adaptación que se apoyó tanto en la selección de los cultivos y aprovechamiento de los 
suelos, como en las prácticas agrarias aplicadas. Un rasgo importante de la producción campesina es su relativo alto grado de autosuficiencia, es decir, que las familias campesinas consumen una parte sustancial de su propia producción y, concomitantemente, producen casi todos los bienes que necesitan (Toledo, 1980). Lo más relevante de la agricultura tradicional es el atraso técnico (escaso o nulo uso de agroquímicos y semilla mejorada) y tecnológico (uso de implementos ancestrales de bajo rendimiento), que implican una economía de subsistencia en la que se consume todo lo que se produce. Esto se traduce en la necesidad de mucha mano de obra y el uso preferente de aperos de labranza no mecánicos (Geografía rural 2007). Hernández (1985: 220), a su vez, nos brinda una explicación más amplia sobre la agricultura tradicional, indicando que ésta se sustenta en «el uso de los recursos naturales basado: a) en una prolongada experiencia empírica que ha conducido a configurar los actuales procesos de producción y las prácticas de manejo utilizadas; b) en un íntimo conocimiento físico-biótico por parte de los productores; c) en la utilización de una educación no formal para transmitir los conocimientos y habilidades requeridas; y d) en un acervo cultural presente en las mentes de la población agrícola».

La productividad de los cultivos, con la agricultura tradicional, está limitada por las características del medio y la disponibilidad de recursos nutritivos; sin embargo, los rendimientos rara vez están por encima de los promedios nacionales, aún en las mejores condiciones (Montalba, 2004). Así como las variedades tradicionales son muy eficientes en condiciones marginales, logrando producir granos (aunque escasamente) con cantidades muy reducidas de nutrientes y humedad, también, por lo general (aunque no siempre), disminuyen fuertemente su eficiencia en condiciones más favorables desde el punto de vista de aumento de nutrientes y humedad (Ibíd.).

Aunque la agricultura tiende a ser la actividad principal de la familia campesina, la subsistencia campesina está basada en una combinación de prácticas, que incluye la recolección agrícola, cuidado de ganado doméstico, artesanía, pesca, caza y trabajos fuera de la explotación a tiempo parcial, estacionales o intermitentes (Toledo, 2004).

\section{MATERIALES Y MÉTODOS}

Es una investigación básica, que contribuye con cierto conocimiento referido a las características de los sistemas de producción agropecuaria tradicionales de Carhuaz, zona de la Cordillera Negra, en el contexto actual y describe las condiciones topográficas y edáfico-climáticas del lugar. De acuerdo al nivel de profundidad es un estudio descriptivo (deductivo), analítico y en algunos aspectos explicativo.

Para el estudio se tomó como unidad de análisis a una familia rural y la muestra estuvo representada por 339 familias, que en forma accidental (casual) fueron encuestadas, partiendo de un considerando predefinido (Tinoco y Sáenz, 1999), es decir, familias que sean encontradas en un lugar visitado en un momento determinado durante el trabajo de campo. Por tanto, se trató de una muestra probabilística, es decir, que todas las familias tuvieron igual probabilidad de ser entrevistadas.

Para la obtención de datos de campo se empleó como instrumento principal la encuesta que se aplicó a los jefes o representantes de las familias integrantes de la muestra. También se entrevistó a líderes y autoridades de los distritos localizados dentro del 
ámbito de estudio. Además se utilizó la técnica de la observación, específicamente para efectuar una descripción de la topografía de la zona y, por último, se tomaron algunas muestras de suelos para conocer la fertilidad referencial de éstos. El análisis de los suelos se realizó en el Laboratorio de Suelos y Aguas de la Facultad de Ciencias Agrarias de la Universidad Nacional «Santiago Antúnez de Mayolo» (UNASAM).

Los datos obtenidos con las encuestas fueron procesados empleando el Programa SPSS versión 21, con el cual se ha obtenido principalmente las frecuencias de ocurrencia y con ello se estableció la predominancia de técnicas y tecnologías empleadas.

\section{RESULTADOS}

1. Caracterización de la topografía, clima y suelos

Los distritos involucrados en el estudio (Yúngar, Anta, Marcará, Carhuaz y Ataquero) abarcan una superficie de 25.530 ha., espacio territorial que se extiende desde los 2600 hasta los $4200 \mathrm{msnm}$. Encierra diferentes condiciones de clima, suelo y vegetación que se manifiestan en la diferenciación vertical de los cultivos que se explotan en esa zona. Por tanto, en función de la altura y clima, se han definido tres zonas bien marcadas: Parte baja, media y alta, cada una con características muy peculiares como a continuación se describen.

1.1 Parte baja. Se extiende entre los 2600 y $3200 \mathrm{msnm}$. En esta zona el clima se caracteriza por presentar una temperatura promedio anual de $15^{\circ} \mathrm{C}$ con precipitaciones del orden de $700 \mathrm{~mm}$ al año. En esta zona se encuentran los mejores suelos por su fertilidad y en ellos, en los últimos 15 años, se viene conduciendo diferentes cultivos comerciales como frutales (palto, lúcumo, lima dulce, melocotón, tuna, etc.), cultivos anuales (papa, maíz, frijol) y forrajes (alfalfa), incluso se observa una cierta especialización en cada distrito.

1.2 Parte media. Se extiende entre los 3200 y $3800 \mathrm{msnm}$, presenta una temperatura promedio anual de $12^{\circ} \mathrm{C}$ y precipitaciones de $750 \mathrm{~mm}$ anuales. Se cultiva cereales menores (trigo, cebada, avena), tubérculos (papa, oca, olluco, mashua) y tarwi.

1.3 Parte alta. Por encima de los $3800 \mathrm{msnm}$, con precipitaciones de 800 a $900 \mathrm{~mm}$. anuales y una temperatura promedio anual menor a $8^{\circ} \mathrm{C}$. Esta zona se encuentra cubierta de pastos nativos para el pastoreo de ganado. Además, es la zona de captación de lluvias. Cabe mencionar que todos los distritos, a excepción de Marcará, tienen parte alta, media y baja.

Otra característica común de la parte baja y media del ámbito de estudio es la presencia de vegetación arbórea y arbustiva, que le brindan una característica peculiar; es decir, árboles en los alrededores de las chacras, de los pastos y a lo largo de la ribera del río Santa. Entre las especies más comunes se tiene el eucalipto (Eucalyptus glóbulus), el molle (Schinus molle), el capulí (Prunus serótina), aliso (Alnus jorulensis), retama (Spartium junceum), etc.

\section{Caracterización técnica de la Producción Agropecuaria Tradicional}

Los sistemas de producción agropecuaria tradicional (PAT) de Carhuaz, a semejanza de otras zonas de la sierra peruana, están constituidos básicamente por tres subsiste- 
mas: el subsistema agrícola (A); el subsistema pecuario (B) y el subsistema forestal (C), los cuales interactúan, de tal manera que se benefician mutuamente; los cultivos son beneficiados por los árboles con materia orgánica, microclima, humedad de los suelos y protección contra la entrada de animales. Los animales domésticos, por su parte, reciben sombra de los árboles para protegerse de la radiación solar y también forraje y, a su vez, generan estiércol para fertilizar los suelos y productos alimenticios para la familia productora (figura 1).

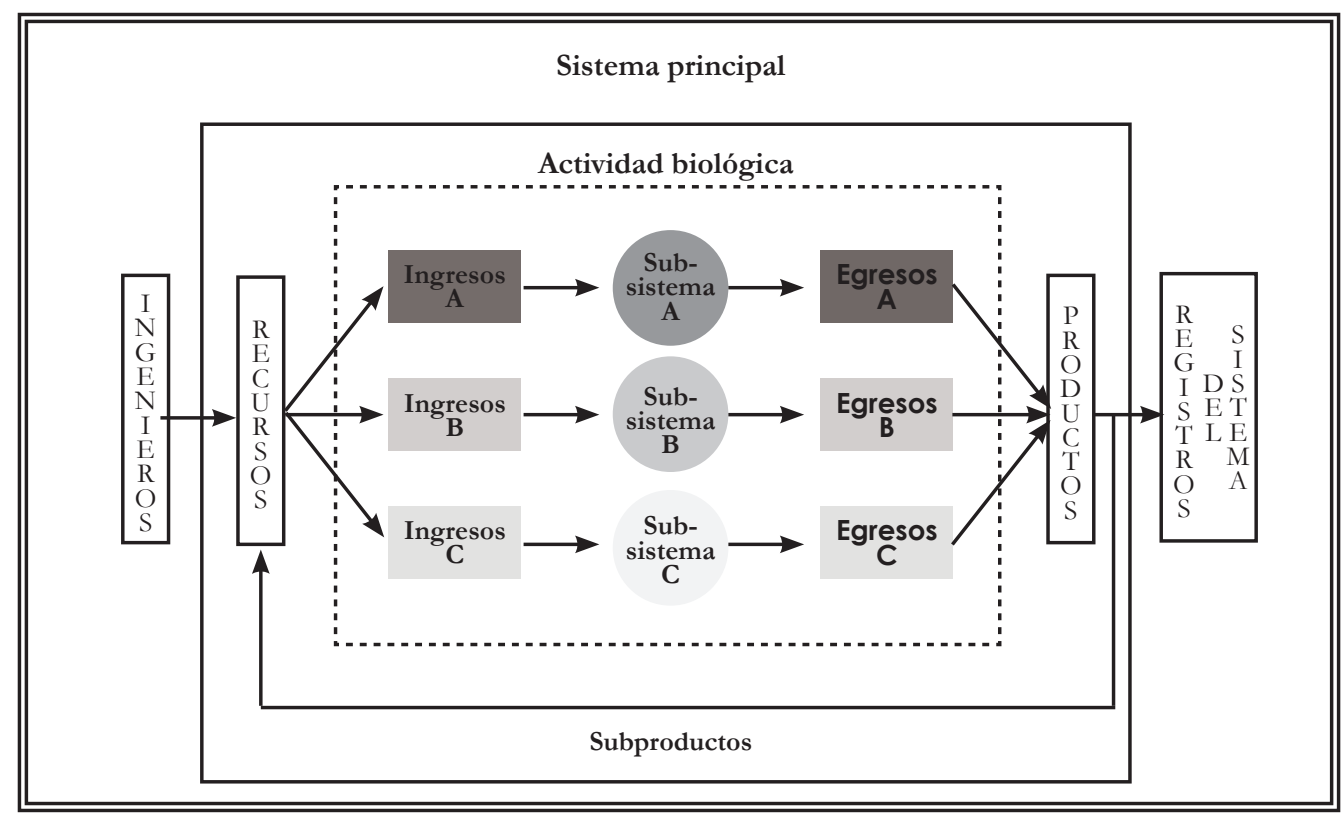

Figura 1. Esquema de un sistema PAT de Carhuaz

Así mismo, el subsistema forestal brinda leña, madera e ingresos complementarios a la familia. La complejidad de las interacciones del sistema hace que éste contribuya decididamente a su estabilidad y resiliencia, controlado y manejado, por supuesto, por la visión y destreza del hombre. Esa es la clave para la sostenibilidad de estos sistemas, a pesar de las inclemencias del clima y de algunos desastres que suelen presentarse.

\subsection{Disponibilidad de terreno y conducción de los cultivos}

Los datos expuestos en la figura 2 nos demuestran que el 16\% de las unidades de producción se ubican como muy pequeñas, pues oscilan entre 0,25 y 0,50 ha., el 49\% de las mismas están entre 0,5 y 1,0 ha; sumando ambos porcentajes tenemos que el $65 \%$ de las chacras tienen una superficie igual o menor a 1 ha. Este hecho refleja la predominancia del minifundio en la zona de estudio. Así mismo, un $27 \%$ de las unidades productivas tiene de 1 a 2 ha y un $8 \%$ de campesinos disponen de más de 2,0 ha.

En lo referente a la conducción de los cultivos, es necesario mencionar que la gran mayoría de los productores campesinos de la zona $(73 \%)$ tienen terrenos bajo riego y en secano, siendo bajo riego solo alrededor del $25 \%$ del total del área de la cual disponen y el resto en secano. Así mismo, hay un 13\% de productores que tienen terrenos solamente bajo riego y un $14 \%$ solo en secano (figura 2). 


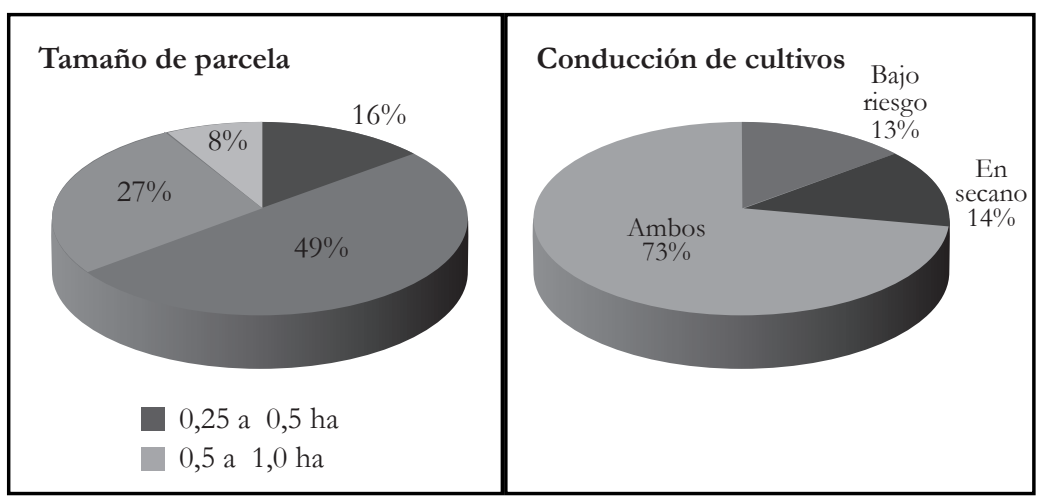

Figura 2. Área de las parcelas y conducción de los cultivos

\subsection{Tecnología empleada}

Semillas utilizadas. En esta zona, para la siembra de sus cultivos, los campesinos utilizan tanto semillas nativas como también mejoradas. En la tabla 1 se puede observar que el mayor porcentaje de semillas utilizadas de los principales cultivos, corresponde a semilla mejorada, como es el caso de papa, trigo, cebada, habas y maíz; en menor medida habas y arveja y tubérculos nativos (con excepción de la papa). En el caso de la alfalfa el 100\% del área sembrada corresponde a semilla mejorada.

Tabla 1. Tipos de semillas empleadas por los campesinos

\begin{tabular}{lccccccccc}
\hline \multicolumn{1}{c}{ Semillas } & Papa & Trigo & Cebada & Habas & Arveja & Chocho & Maíz & Alfalfa & Oca \\
\hline Nativas & 2,9 & 5,7 & 4,6 & 8,6 & 2,90 & 1,10 & 12,50 & 0,00 & 7,2 \\
Mejorada & 38,6 & 67,5 & 39,6 & 20,7 & 18,20 & 0,0 & 47,90 & 17,10 & 0,0 \\
Ambos & 28,9 & -- & -- & 0,4 & 0,40 & -- & -- & -- & 0,7 \\
No siembra & 29,6 & 26,8 & 55,7 & 70,4 & 78,60 & 98,9 & 37,50 & 82,90 & 92,1 \\
\hline
\end{tabular}

Empleo de Fertilizantes Minerales. En cuanto al uso de fertilizantes minerales, los resultados del estudio demuestran que éstos son utilizados prioritariamente para los cultivos comerciales como papa, quinua, maíz, oca y alfalfa, empleándose muy poco en trigo, cebada, habas y arveja y nada en chocho (figura 3).

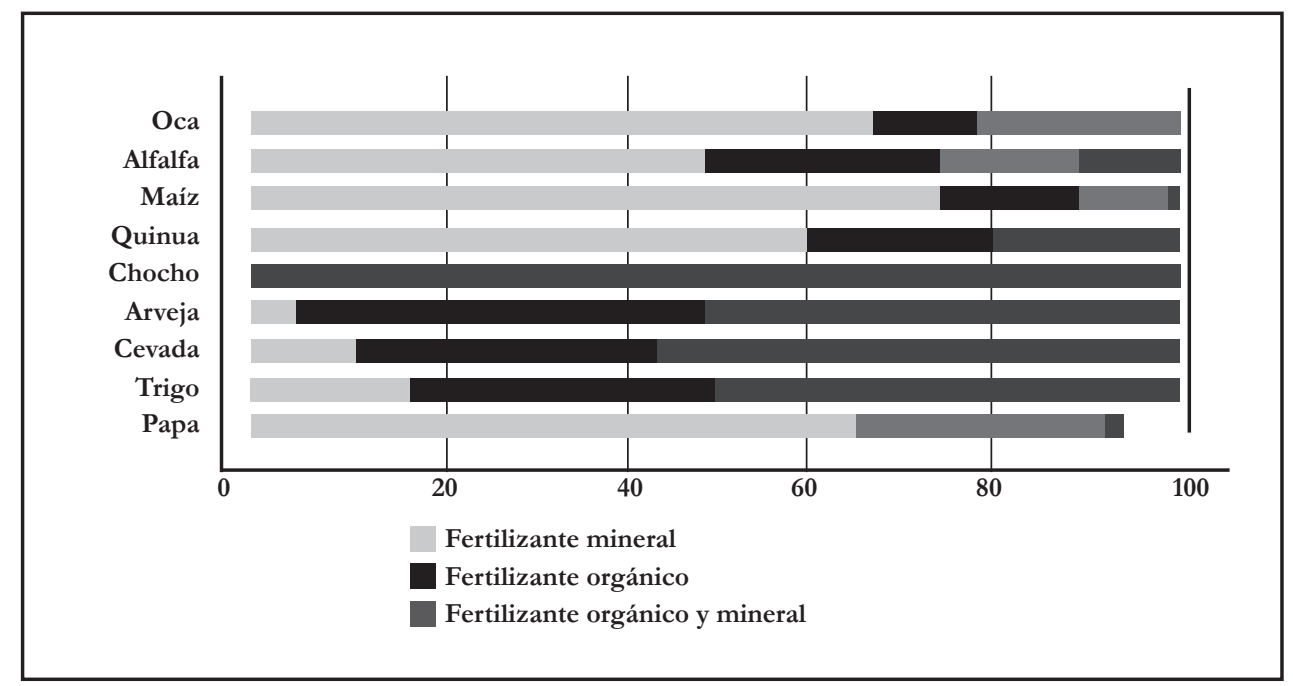

Figura 3. Empleo de fertilizantes minerales 
En cuanto a la dosis de fertilización empleada, la encuesta demuestra que el 54,3\% de los campesinos emplean dosis bajas; un 41,4\% dosis medias y solo un 4,3\% aplican dosis altas de fertilización (tabla 3), hecho que está relacionado con la falta de recursos económicos y el gran temor al riesgo de invertir.

Tabla 2. Dosis de fertilización empleadas en la zona

\begin{tabular}{cc}
\hline Dosis de fertilización & Porcentaje \\
\hline Alta & 4,3 \\
Media & 41,4 \\
Baja & 54,3 \\
Total & 100,0 \\
\hline
\end{tabular}

\subsection{Fenómenos adversos de la producción agrícola}

En la zona de estudio se presenta una serie de fenómenos adversos que, en cierta medida, limitan la producción y los rendimientos de los diferentes cultivos, entre ellos tenemos: las heladas, granizadas, sequías, las plagas y enfermedades.

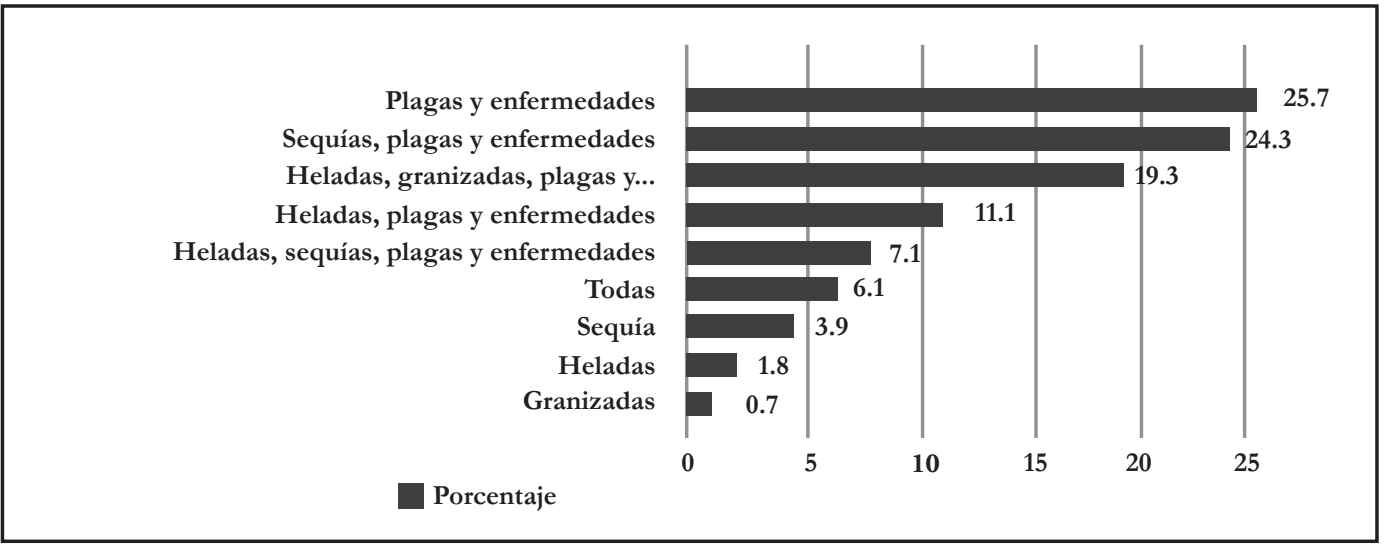

Figura 4. Fenómenos adversos para la producción agrícola

En la figura 4 se puede observar que los campesinos dan mayor importancia a las plagas y enfermedades (25,7\%); luego están las sequías conjuntamente con las plagas y enfermedades $(24,3 \%)$, enseguida está la presencia conjunta de heladas, granizadas y plagas y enfermedades $(19,3 \%)$, también heladas con plagas y enfermedades $(11,1 \%)$, menor importancia tienen las granizadas por no ser tan frecuentes e incluso hay años en que no ocurren.

\subsection{Tipos de crianzas en la zona}

Uno de los complementos más importantes de la economía campesina es la crianza de animales domésticos. Dentro de los diferentes tipos de crianzas, el cuy ocupa un lugar privilegiado, el 93,2\% de los campesinos cría cuyes, el segundo lugar lo ocupan las aves (83,9\%); luego están los porcinos con 70,3\%. También está el ganado ovino con $53,8 \%$; los equinos (caballos y burros) con 40,5\%; en mucho menor cantidad los vacunos $(28,7 \%)$ y por último están los conejos con solo $5 \%$ (figura 5 ). 


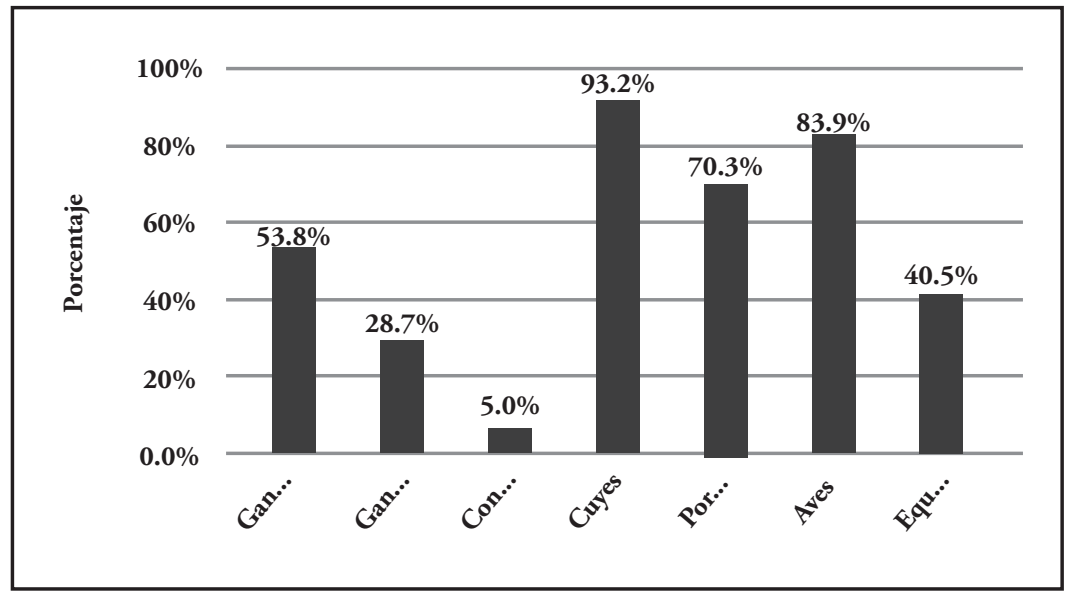

Figura 5. Tipos de animales domésticos en la zona de estudio

\section{DISCUSIÓN}

En el ámbito de estudio predomina el minifundio que imprime una característica muy peculiar, ya que en parcelas muy pequeñas se ve limitada la aplicación de alta tecnología o el empleo de maquinaria para la preparación de los suelos, por lo que la mayoría lo hace utilizando el arado jalado por bueyes o simplemente la realización manual de las diferentes labores culturales y, por ello, el rendimiento de los cultivos es bajo. Más aún, la mayoría de predios se encuentra en laderas con diferentes pendientes, los suelos son poco profundos y en algunos casos pedregosos. Esto concuerda con Echenique (2006) quien, en un trabajo realizado en varios países de Latinoamérica, reporta que los predios de la agricultura familiar se ubican como los más pequeños de la tenencia agrícola y se ubican generalmente en áreas con menor potencial de recursos (suelo y agua).

A este respecto, Alva (2010: 1) afirma que, «el pequeño productor rural no es considerado como un actor importante del desarrollo rural del país y de las regiones y continúa afirmando, que en el fondo existe la tesis de la inviabilidad de la pequeña agricultura bajo la condena al minifundio, la dispersión parcelaria y la tradicionalidad. Consecuentemente, la apuesta ha sido por la gran propiedad agraria, y en particular por aquella que se condice con la actividad agroexportadora».

García (2009) a su vez, al realizar un análisis crítico de la Reforma Agraria de México mencionaba que el minifundio implica subempleo, ya que al no poder obtener de la tierra el ingreso necesario para sobrevivir, se ve obligado no solo a vender su fuerza de trabajo y seguir cultivando su parcela, sino también a emigrar y abandonarla o venderla. Así mismo, la conducción de los cultivos en secano conlleva riesgos, habida cuenta que la producción estará supeditada a la presencia o ausencia de las lluvias. Por eso, los campesinos siempre tienen cultivos en secano y bajo riego para asegurar la obtención de productos para su alimentación; en caso contrario tratan de dedicarse a la crianza de animales domésticos o vender su mano de obra o algún otro servicio.

En lo referente a la tecnología empleada, se ha encontrado que, actualmente, hay una tendencia a utilizar cada vez más las variedades mejoradas, sobre todo por los llamados «productores progresistas»; es decir, aquellos que están en permanente búsqueda de algo nuevo para mejorar sus rendimientos. Sin embargo, aún se sigue utilizando un pequeño porcentaje de semilla nativa para la producción de algunos cultivos, 
especialmente en papa, habas, oca y maíz. En el caso de la alfalfa, el 100\% de los productores han pasado al uso de semilla mejorada, por ser variedades más precoces y más productivas y esa tendencia se ve seguir en la mayoría de los cultivos a excepción de los tubérculos andinos (papa, oca, olluco, mashua, etc.), los cuales, al parecer, mantendrán el uso de la semilla nativa, ya que les permite obtener productos de calidad y ser competitivos en el mercado nacional e internacional.

En cuanto al uso de fertilizantes minerales, el campesino es consciente que para poder competir en el mercado necesita, por un lado obtener mayores rendimientos y, por otro, obtener productos de mejor calidad y que eso se logra con tecnología, es decir, suministrando a la planta las cantidades adecuadas de nutrientes, mediante el uso de fertilizantes, paralelamente al uso de semillas mejoradas y el control oportuno de plagas y enfermedades. Con la apertura de los mercados y la inserción del campesino a ellos, en esta zona del país, se vislumbra cambios importantes referidos a la tecnología de producción agrícola. Al parecer la tendencia es al uso de semillas mejoradas y al uso intensivo de fertilizantes y plaguicidas. El cambio es gradual, por el momento son los campesinos de la parte baja (entre los 2600 y $3200 \mathrm{msnm}$ ), los que tratan de modernizar los procesos de producción, a fin de obtener mejores rendimientos y con ello poder competir en el mercado. En la parte media (entre los 3200 y $3800 \mathrm{msnm}$ ) la población mantiene las variedades nativas y hace poco uso de fertilizantes minerales, esto se debe a que, en esta parte, los campesinos tienen menores ingresos y, por tanto, un gran temor al riesgo de perder su inversión. Al respecto, Montalba (2004) menciona que las variedades tradicionales son muy eficientes en condiciones marginales, logrando producir granos (aunque escasamente) con cantidades muy reducidas de nutrientes y humedad; también, por lo general (aunque no siempre), disminuyen fuertemente su eficiencia en condiciones más favorables (desde el punto de vista de aumento de nutrientes y humedad).

Referente a la dosis de fertilización, predominan las dosis bajas, hecho que está relacionado con el temor al riesgo de invertir en insumos, perder la cosecha y no recuperar lo invertido. Además, los campesinos minifundistas generalmente se encuentran en pobreza y no tienen los medios económicos suficientes para invertir en agroquímicos, a pesar de ser conscientes de que la aplicación de fertilizantes contribuye a mejorar los rendimientos y la calidad de las cosechas.

En la zona de estudio se presenta una serie de fenómenos adversos que, en cierta medida, limitan la producción y los rendimientos de los diferentes cultivos, entre ellos tenemos: las heladas, granizadas, sequías y las plagas y enfermedades. Sin embargo, estos eventos pueden presentarse por separado o en forma conjunta dos o más fenómenos y, por eso, los campesinos les dan la importancia de diferente manera, de acuerdo al distrito y a la altura sobre el nivel del mar. Estos fenómenos se pueden considerar como factores limitantes de la producción. Paralelamente a los factores limitantes, existen otros problemas que afectan la producción como son: la topografía quebrada del terreno, el minifundio y la baja disponibilidad de agua de riego, es decir, problemas que no pueden ser solucionados por los campesinos. Sin embargo, ellos a través de sus dirigentes tratan de presentar propuestas para incrementar la disponibilidad de agua de riego, ya que el recurso hídrico es escaso y, al mismo tiempo, limitante de la producción. 
En el ámbito de estudio hay diferentes tipos de animales domésticos y todos ellos de importancia para el trabajo y la economía del campesino. Los animales proveen carne, leche y huevos para la alimentación de la población; pero también lana para las artesanías, y fuerza de trabajo como apoyo a los productores (bueyes y equinos). Es decir, las crianzas constituyen un componente muy importante de la economía campesina. Los cuyes son la especie más difundida por ser animales de rápido crecimiento y propagación. Bajo condiciones de manejo óptimo, en el transcurso de 1 año la cantidad de cuyes puede incrementarse hasta 10 veces y por eso, requiere manejo técnico, sacas y descartes oportunos y la alimentación controlada. El 93\% de la población cría cuyes y periódicamente se deleita consumiendo su carne. Los cuyes tienen gran demanda en el mercado local, regional y nacional e incluso se están exportando al extranjero. Algunas familias se dedican exclusivamente a la crianza de cuyes en forma empresarial, manejando 600 a 800 cabezas y obtienen buenos ingresos anuales. Los cuyes son los animales más rústicos que los campesinos crían y, al mismo tiempo, los que les brindan proteínas de origen animal para la alimentación cotidiana e ingresos periódicos, para la compra de aquellos productos o bienes que no producen. Cabe mencionar que el campesino, sobre todo el de la parte media (3200 - $3800 \mathrm{msnm}$ ), consciente de las condiciones del medio donde vive, es una persona muy previsora, pues trata de asegurar la alimentación familiar sobre la base de la siembra de varios cultivos (policultivos), la crianza de varios tipos de animales y la realización de otras actividades complementarias. La tecnología tradicional indígena y campesina se ubica en una concepción amplia del universo y de la sociedad, que parte de la tierra como base de todos los derechos humanos individuales y sociales, como el principal elemento de subsistencia de la sociedad (Gómez et al., 1998).

Algunos animales domésticos son utilizados como apoyo en la producción de los cultivos. Por ejemplo, crían bueyes para el arado o preparación del terreno durante la época de siembra de los cultivos y al mismo tiempo obtener ingresos complementarios por el alquiler de los bueyes a sus vecinos; los burros y caballos son utilizados para el transporte de sus cosechas del campo a su casa o de la casa hacia las ferias y mercados locales. Con ello, los campesinos logran básicamente la autosuficiencia de la actividad agropecuaria. Esto concuerda con Toledo (2004:7), quien menciona que «aunque la agricultura tiende a ser la actividad principal de la familia campesina, la subsistencia campesina está basada en una combinación de actividades, que incluyen la recolección agrícola, cuidado de ganado doméstico, artesanías, pesca, caza y trabajos fuera de la explotación a tiempo parcial, estacionales o intermitentes».

\section{CONCLUSIONES}

El ámbito de estudio que involucra los distritos de Yúngar, Anta, parte de Marcará, Carhuaz y Ataquero, abarca una superficie de 25,530 ha.; está localizado en la Cordillera Negra entre los 2600 y 4200 msnm, tiene una topografía accidentada, presenta una diferenciación vertical de clima, suelos y vegetación, habiéndose definido tres zonas bien marcadas: parte baja, media y alta, cada una de las cuales tiene condiciones climáticas, topográficas y de suelo muy particulares que determinan los sistemas de cultivos y crianzas. 
Los sistemas de producción tradicionales, en la zona de estudio, se desarrollan en parcelas muy pequeñas, el 55\% de los productores tiene predios con menos de 1 ha. y además los cultivos se conducen básicamente en secano; solo un pequeño porcentaje se conduce bajo riego, siendo escasa la disponibilidad de agua de riego.

Los productores emplean, para la producción agrícola, mayormente semilla mejorada, ha disminuido notoriamente el uso de semilla nativa. Además se utiliza fertilizantes y pesticidas solamente para algunos cultivos, especialmente para los cultivos comerciales (papa, maíz, frutales diversos y alfalfa), el resto se conduce sin agroquímicos, solamente con guano de corral.

Los campesinos crían diferentes tipos de animales como componente importante de la economía campesina, y son los cuyes los más apreciados por su rápido crecimiento, importancia nutricional y demanda en el mercado.

En el sistema de producción agropecuaria tradicional interactúan armónicamente la agricultura, las crianzas y la explotación forestal, beneficiándose mutuamente para generar mayores rendimientos y mejoras a las familias gestoras.

\section{AGRADECIMIENTOS}

El especial reconocimiento a los líderes y autoridades del ámbito de estudio, por su contribución con información y facilidades para la aplicación de encuestas en el ámbito de trabajo.

\section{REFERENCIAS BIBLIOGRÁFICAS}

Alva, Pedro. 2010. Encuentro regional «Pequeña propiedad y desarrollo agrario», realizado en Lambayeque el 23 de junio del 2010 y publicado en el Semanario Expresión N ${ }^{\circ}$ 701, en Febrero 2011. Blog «Pequeña Agricultura y Desarrollo Rural». <http:// blog.pucp.edu.pe/item/123610/pequena-agricultura-y-desarrollo-rural $>$ [Consulta: 02-02-2015].

Echenique, Jorge. 2006. Caracterización de la Agricultura Familiar. Documento preparado para la Oficina Regional de FAO para América Latina y el Caribe y el BID. Proyecto GCP-RLA-152-IAB Bloque Comercio FAO/BID. 15.

García, Fernando. 2009. El papel del minifundio en el desarrollo agrícola de México. Análisis del medio rural Latinoamericano. <http://www.google.com.pe/ url? sa $=\mathrm{t} \& \mathrm{rct}=\mathrm{j} \& \mathrm{q}=\& \mathrm{esrc}=\mathrm{s} \&$ source $=$ web\&cd $=2 \& \mathrm{ved}=0 \mathrm{CCAQFjA}>$ [Consulta: 12-04-2015].

Geografía Rural. 2007. Los sistemas agrarios tradicionales. <http://geografia.laguia2007. $\mathrm{com} /$ geografia-rural/sistemas-agrarios-tradicionales $>$ [Consulta: 15-11-2012].

Gómez, Gerardo; Ruíz, José y Bravo, Salvador. 1998. Tecnología Tradicional Indígena y la Conservación de los Recursos Naturales. Conferencia presentada en el Encuentro Latinoamericano sobre Derechos humanos y Pueblos Indios, Universidad San Carlos. <http://biblio.juridicas.unam.mx/libros/1/99/11.pdf> [Consulta: 2812-2014]. 
Hernández, Efraim. 1985. Agricultura Tradicionaly desarrollo: Qué es la tecnología Tradicional? <http://www.laneta. apc.org/pasos/fxolo3.htm> [Consulta: 14-05-2015].

Montalba, René. 2004. Historia de la transformación del sistema hombre en el medioambiente en el secano interior de la IX Región de Chile. Una aproximación agroecológica. Revista CUHUSO (Chile). Volumen 8. 18-38.

Naredo, José. 1996. Sobre la reposición natural y artificial de agua y de nutrientes en los sistemas agrarios y las dificultades que comporta su medición y seguimiento. En: La fertilización en los sistemas agrarios, una perspectiva histórica. R Garrabou y J. M. Naredo (editores). Fundación Argentaria. Madrid, España. 17-34.

Tinoco, Zaira y Sáenz, Dora. 1999. Investigación científica. Protocolo de Investigación. Rev. Fármacos, 1999, Vol. 12, No 1: 78-101.

Toledo, Víctor. 2004. La Racionalidad Ecológica de la Producción Campesina. Centro Latino de Desarrollo, CLADE. Universidad Nacional de México. 12.

Toledo, Víctor. 1980. La Ecología del modo campesino de producción, Antropología y Marxismo, Vol. No 3. México. 35-55.

Fecha de recepción: 15 de enero de 2015

Fecha de aceptación: 10 de marzo de 2015

\section{Correspondencia}

Juan Barreto Rodríguez

jubaguez@hotmail.com 